\title{
Demografia de Geonoma gamiova em área de extrativismo foliar
}

\author{
Geonoma gamiova demography in an area with leaf harvesting
}

\section{Marilia de Fátima Ceccon-Valente ${ }^{\mathrm{I}}$ Raquel Rejane Bonato Negrelle $^{\mathrm{I}}$}

\section{RESUMO}

As folhas de Geonoma gamiova Barb. Rodr. são importantes recursos para floriculturas, já que são empregradas principalmente nos arranjos fúnebres. Visando a gerar subsídios para o seu extrativismo sustentável, são apresentados resultados de estudo de estrutura populacional dessa espécie. A pesquisa foi realizada em área de Floresta Ombrófila Densa Submontana onde a espécie é submetida ao extrativismo foliar (Comunidade do Rasgadinho, Mun. Guaratuba, Paraná, 25 45'S e 48 46'W, 30 - 100m de altitude). Detectou-se alta densidade de indivíduos, principalmente nas classes mais jovens, caracterizando um padrão populacional J-invertido. Registrou-se estoque de 448 folhas aptas para comercialização em 0,5ha, com histórico de intensa extração de folhas. Evidenciou-se alto potencial de regeneração e padrão de distribuição agregado (Id>1) para a população estudada. Com base nos dados obtidos, discutiu-se a sustentabilidade da atividade extrativista em questão.

Palavras-chave: planta ornamental, palmeira, floricultura, manejo florestal.

\section{ABSTRACT}

The leaves of Geonoma gamiova Barb. Rodr. are an important resource to floriculture, where they are used specially in funeral floral ornaments. Aiming to generate subsidies to sustainable management of this resource, results from the evaluation of the population structure of this species are presented. The research was performed in Sub-Montane Atlantic forest submitted to leaf harvesting of G. gamiova (Rasgadinho community, Guaratuba Mun., Paraná Brasil; 2545 'S e 4846 'W). It was registered high density of this species, mainly in younger stages, characterizing a J-inverted population. It was found 448 leaves commercially acceptable in the 0.5ha plot with a history of intense leaf harvesting. The population showed a high potential of regeneration and clustered spatial distribution (Id $>1)$. Based on these data, it is discussed the sustainability of the cited harvesting activity.

Key words: ornamental plant, palms, floriculture, forest management.

\section{INTRODUÇÃO}

Geonoma gamiova Barb. Rodr. (Arecaceae) é uma palmeira de estipes múltiplos formando touceira típica do sub-bosque das encostas da Floresta Atlântica na Costa Sudeste do Brasil, em altitudes menores que $800 \mathrm{~m}$, ou raramente até $1.300 \mathrm{~m}$ (HENDERSON et al., 1997). Devido classificação em espécie seletiva ciófita (REITZ, 1974), é somente constatada nas matas em bom estado de conservação. É uma espécie de hábito arbustivo, com estipes simples e anelados de 2 a $4 \mathrm{~m}$ de altura, em geral, com $3 \mathrm{~cm}$ de diâmetro, agrupados em touceiras de três ou mais estipes. Cada estipe apresenta em média oito a 15 folhas, com a lâmina variando entre 50 a $80 \mathrm{~cm}$ de comprimento (REITZ, 1974).

As palmeiras são reconhecidas fontes de produtos florestais não-madeiráveis (PFNMs). Entre seus diversos recursos, destacam-se o palmito, os frutos e as folhas, que podem ser utilizados para alimentação, moradia, artesanato, vestimenta, entre outros usos (FAO, 1992 e 1995; MOUSSOURIS \& REGATO, 1999). Na região do litoral do Paraná, a palmeira Geonoma gamiova, popularmente chamada de palha, é considerada um importante PFNM utilizado pela população rural da APA de Guaratuba (SONDA, 2002; BALZON, 2006). Inicialmente, suas folhas eram utilizadas para cobertura das moradias dos residentes locais. Atualmente, registra-se intenso extrativismo das folhas dessa espécie, que atende demanda de floriculturas da capital do estado e

\footnotetext{
'Laboratório OIKOS, Departamento de Botânica, Universidade Federal do Paraná (UFPR), CP 19031, 80610-020, Curitiba, PR, Brasil.
} E-mail: negrelle@ufpr.br.*Autor para correspondência. 
outros centros urbanos, as quais são empregadas principalmente na confecção de arranjos fúnebres (CECCON-VALENTE, 2009).

Para que PFNMs como a palha (G. gamiova) sejam reconhecidos como instrumentos de alta potencialidade para o desenvolvimento sustentável de comunidades rurais e preservação dos ambientes de origem desse recurso, as lacunas no conhecimento dos limites ecológicos do extrativismo devem ser preenchidas. Estudos sobre a autoecologia de outras espécies fonte de PFNMs indicam a relevância de se investigar a demografia e a história de vida dessas espécies (SVENNING \& MACIA, 2002; RODRIGUEZ-BURITICÁ et al., 2005; BAULDAUF, 2006; SCHIMIDT et al., 2007).

Mesmo com histórico de uso de muitos anos no litoral do Paraná, praticamente não existe informação sobre a ecologia básica de G. gamiova capaz de subsidiar a sustentabilidade da prática extrativista atual. Por ser um recurso fundamental para o desenvolvimento econômico e social da região rural do litoral do Estado, e existir uma preocupação com a preservação do bioma onde está inserida, torna-se crucial levantar informações botânicas e ecológicas sobre a espécie para que sua exploração possa ser ecologicamente viável. Nesse contexto, apresentam-se resultados de estudo que visou a: a) caracterizar a estrutura populacional de G. gamiova em área submetida ao extrativismo foliar e analisar comparativamente os padrões encontrados em estudos com outras espécies do mesmo gênero; b) avaliar a expectativa do nicho de regeneração vir a participar do componente adulto da população em estudo; c) analisar o estoque de recurso comercialmente disponível na área amostral. Com base nos dados obtidos, discutiu-se a sustentabilidade da atividade extrativista em questão.

\section{MATERIAL E MÉTODOS}

A população de $\boldsymbol{G}$. gamiova estudada localizava-se na comunidade do Rasgadinho, zona rural do município de Guaratuba-PR ( 25 45'S e 48 $46^{\prime} \mathrm{W}, 30$ - 100m de altitude). O clima da região escarpada da APA de Guaratuba, onde está inserida a comunidade do Rasgadinho é do tipo Cfa, descrito como clima subtropical úmido com verão quente, segundo a classificação de Köppen. O mês mais quente possui temperatura média superior a $22^{\circ} \mathrm{C}$ e o mais frio mostra temperatura média inferior a $18^{\circ} \mathrm{C}$, constantemente úmido. As chuvas são regulares no decorrer do ano, não apresentando estação seca (IAPAR, 2000). A vegetação encontra-se no domínio da Floresta Ombrófila Densa Submontana (VELOSO et al., 1991). Essa formação do Bioma Mata Atlântica ocupa, para a latitude do local (entre os paralelos $24^{\circ}$ e $32^{\circ} \mathrm{S}$ ), as encostas desde 30 a $400 \mathrm{~m}$ de altitude. Assim, a cobertura típica é florestal, multiestratificada e altamente diversificada, com um dossel que pode atingir 30 ou até $35 \mathrm{~m}$ (RODERJAN et al., 2002).

Para realização deste estudo, em julho de 2008, foi demarcada área amostral de 0,5ha, subdividida em 50 parcelas de $100 \mathrm{~m}^{2}$ em local de contínua ação extrativista pela comunidade, há cerca de 20 anos. Para a caracterização da estrutura da população, foram coletados dados referentes à altura e estádio de desenvolvimento de cada indivíduo da área amostral. Para a amostragem das plântulas, foram selecionadas cinco parcelas aleatoriamente, totalizando $10 \%$ da área $(0,05 \mathrm{ha})$. Por ser uma palmeira de estipes múltiplos, considerou-se a touceira como indivíduo e a cada uma das suas ramificações denominou-se estipe. Os estipes foram classificados nos seguintes estádios de desenvolvimento, baseados na altura e características morfológicas: Plântulas estipes individuais ou em touceiras, com folhas não pinadas e sem estipe exposto; Jovens - estipes com até $1 \mathrm{~m}$ de altura, geralmente em touceiras, com pelo menos uma folha pinada, com ou sem estipe exposto; Adultos não-reprodutivos - estipes com altura superior a $1 \mathrm{~m}$, com estipe exposto e sem evidência de atividade reprodutiva, geralmente em touceiras; Adultos reprodutivos - estipes com evidência de atividade reprodutiva, geralmente em touceiras. Essas touceiras foram classificadas de acordo ao estádio do estipe em estádio mais avançado de desenvolvimento, podendo apresentar estipes de todas as outras classes anteriores a esta.

A distribuição espacial da população foi avaliada a partir das coordenadas espaciais (x;y) de cada indivíduo e cálculo do Índice de Morisita (Id), considerando-se: $\mathrm{Id}=1$, distribuição aleatória; $\mathrm{Id}>1$, distribuição agrupada; Id $<1$, distribuição uniforme. Aplicou-se o teste de significância de Id utilizando o teste Qui-quadrado (KREBS, 1989).

Para verificar se as classes que compõem o nicho de regeneração viriam a participar do componente adulto da população em estudo, avaliouse o Potencial de Regeneração Natural (PRN) da população, de acordo a NEGRELLE (1995). O PRN da espécie foi obtido pela soma dos valores do potencial de regeneração das classes etárias consideradas $\left(\mathrm{PRN}=\mathrm{PR}_{\text {plântula }}+\mathrm{PR}_{\text {jovem }}+\mathrm{PR}_{\text {imaturo }}\right)$.

$\mathrm{O}$ cálculo do potencial de regeneração das distintas classes etárias $\left(\mathrm{PR}_{\mathrm{x}}\right)$ teve como base a densidade relativa (DR) e a frequência relativa (FR) 
de plântulas, jovens e adultos não-reprodutivos, conforme detalhado a seguir:

$$
\begin{aligned}
& \mathrm{PR}_{\mathrm{x}}=\left(\mathrm{DR}_{\mathrm{x}}+\mathrm{FR}_{\mathrm{x}}\right) / 2 ; \\
& \mathrm{DR}_{\mathrm{x}}=\left(\mathrm{DA}_{\mathrm{x}} / \mathrm{N}\right) \mathrm{x} 100, \text { sendo } \mathrm{N}=\text { número }
\end{aligned}
$$

total de indivíduos amostrados;

$\mathrm{DA}_{\mathrm{x}}=\mathrm{N}_{\mathrm{x}}$ sendo $\mathrm{N}_{\mathrm{x}}=$ número total de indivíduos amostrados na classe etária $\mathrm{x}$;

$\mathrm{FR}_{\mathrm{x}}=\left(\mathrm{FA}_{\mathrm{x} /} \Sigma \mathrm{FA}\right) \mathrm{x} 100, \quad$ sendo $\quad \Sigma \mathrm{FA}=$ somatório das frequências absolutas de todas as classes etárias;

$\mathrm{FA}_{\mathrm{x}}=\left(\mathrm{P}_{\mathrm{x}} / \mathrm{P}_{\mathrm{t}}\right) \mathrm{x} 100$, sendo $\mathrm{P}_{\mathrm{x}}=$ número de parcelas em que a classe etária $\mathrm{x}$ foi registrada, $\mathrm{P}_{\mathrm{t}}=$ número total de parcelas $=50$.

Para mensurar e analisar o estoque de recurso comercialmente disponível, foram coletados dados referentes ao número de folhas em ponto de corte de todos os estipes dos indivíduos da área amostral, exceto plântulas, e quantificado o número de estipes com sinais de extração foliar em cada classe. Segundo o método de coleta dos extratores da comunidade, as folhas em ponto de corte apresentam no mínimo $30 \mathrm{~cm}$ de comprimento (desde a abertura do primeiro folíolo até o término do último) e devem estar livres de fungos ou danos na superfície. Os estipes com danos de extrativismo eram facilmente reconhecidos pela permanência dos pecíolos com sinal de corte.

\section{RESULTADOS E DISCUSSÕES}

A densidade populacional de Geonoma gamiova foi de 6.578 touceiras (8.318 estipes) por hectare na área de estudo. A maior parte das touceiras de G. gamiova encontrava-se nos estádios iniciais de desenvolvimento:jovem (27\%) e plântula (67\%), o que caracteriza uma população com curva de distribuição das classes em "J" invertido ou exponencial negativa (Tabela 1). Todas as classes etárias apresentaram padrão de distribuição agregado (Id>1) (Tabela 2), com ocorrência em toda a área amostral.
O Potencial de regeneração natural encontrado para os indivíduos da classe plântula foi de 49\%, ou seja, os indivíduos dessa classe apresentavam alta probabilidade de virem a participar do componente adulto. Contudo, comparando-se os valores de densidade registrados para plântulas e adultos reprodutivos, evidenciou-se alta taxa de mortalidade, sendo este processo também evidenciado pela diminuição do Potencial de Regeneração natural nos estádios mais avançados de desenvolvimento dos indivíduos (Tabela 1).

Foram registradas 448 folhas em ponto de corte, ou seja, comercialmente disponíveis na área amostral. A maioria destas foi registrada nos estipes jovens $(91 \%, n=409)$, com menor incidência nos estipes adultos não-reprodutivos $(7 \%, \mathrm{n}=31) \mathrm{e}$ reprodutivos $(2 \%, n=8)$.

Do total de estipes amostrados, exceto plântulas, $28 \%$ apresentou sinal de extração. Dentre estes, os estipes com maior taxa de sinal de extração foram os adultos reprodutivos (74\%), seguido pelos adultos não-reprodutivos $(60 \%)$ e jovens $(22 \%)$. No entanto, apesar de apresentarem menor porcentagem, em números absolutos, a classe jovem possuía a maior quantidade de estipes com sinal de extrativismo.

Em geral, as espécies do gênero Geonoma são naturalmente muito abundantes no sub-bosque de florestas tropicais (RODRIGUEZ-BURITICÁ et al., 2005; SOUZA \& MARTINS, 2006). A densidade de G. gamiova na área de estudo está muito próxima do encontrado para outras espécies sob pressão ou não de extrativismo (SVENNING \& MACIA, 2002; RODRIGUEZ-BURITICÁ et al., 2005; SOUZA \& MARTINS, 2006). Estudos conduzidos com espécies cujas folhas também são extraídas, indicam que esse grupo tem um grande potencial para ser fonte de PFNM, devido à alta densidade. Porém, é necessário que seja realizado um manejo apropriado respeitando a dinâmica de regeneração natural da espécie e dos ecossistemas em que está inserida (SVENNING \& MACIA, 2002; RODRIGUEZ-BURITICÁ et al., 2005).

Tabela 1 - Densidade absoluta (DA), densidade relativa (DR), frequência absoluta (FA), frequência relativa (FR) e potencial de regeneração natural de indivíduos da população de Geonoma gamiova (0,5ha, Comunidade de Rasgadinho, Mun. Guaratuba, PR).

\begin{tabular}{lllcrr}
\hline & DA & DR & FA(\%) & FR(\%) & PRN(\%) \\
\hline Adultos reprodutivos & 49 & 0,01 & 54 & 16 & 24 \\
Adultos não-reprodutivos & 136 & 0,04 & 80 & 30 & 29 \\
Jovens & 894 & 0,27 & 100 & 30 & 49 \\
Plântulas & $2.210^{*}$ & 0,67 & 100 & 30 \\
\hline
\end{tabular}

*valor extrapolado a partir de amostragem em 0,05 ha. 
Tabela 2 - Índice de dispersão (Id) e respectivo teste de significância registrado para população de Geonoma gamiova $(0,5$ ha, Comunidade de Rasgadinho, Mun. Guaratuba, PR). ( $\mathrm{n}=$ número de indivíduos, $x^{2}=$ valor de qui-quadrado) $* \mathrm{P}<0,05$, $\mathrm{gl}=49, * * \mathrm{P}<0,05, \mathrm{gl}=4$.

\begin{tabular}{llcc}
\hline Classe & $\mathrm{n}$ & $\mathrm{Id}$ & $x^{2}$ \\
\hline Adultos reprodutivos & 49 & 1,53 & $74,469^{*}$ \\
Adultos não-reprodutivos & 136 & 1,26 & $83,853^{*}$ \\
Jovens & 894 & 1,18 & $211,928^{*}$ \\
Plântulas & 221 & 1,11 & $27,303 * *$ \\
\hline
\end{tabular}

O padrão de distribuição diamétrica em $\mathrm{J}$ invertido registrado para a população estudada caracteriza-se pela diminuição da frequência, à medida que aumenta o tamanho da classe (SCOLFORO et al., 1998) e sugere que uma população estável e auto-regenerativa, havendo um equilíbrio entre mortalidade e recrutamento dos indivíduos. Padrões de crescimento inicial lentos também podem resultar em distribuições na forma de $\mathrm{J}$ invertido, devido à permanência dos indivíduos por longo período de tempo na mesma classe (WEBB et al., 1972).

Adistribuição espacial agregada encontrada na população estudada também é descrita para outras palmeiras clonais (BAROT et al., 1999, SVENNING, 2001). Essa característica, associada à alta densidade, pode subsidiar plantios e repovoamentos da espécie na área de estudo, dado que, para permitir a viabilidade econômica extrativa, sem prejudicar as demandas crescentes de mercado e causar impacto negativo ao ambiente, há necessidade de conservação da espécie, bem como a domesticação e o estabelecimento de unidades de cultivo (HOMMA, 2008).

No entanto, mesmo em situação de cultivo, o impacto causado pelo extrativismo é geralmente difícil de ser avaliado em nível populacional, visto que não é um processo destrutivo para os indivíduos. Em geral, as consequências da retirada de folhas de palmeiras de sub-bosque têm sido estudadas a nível fisiológico ou em respostas individuais. O corte de folhas em geral estimula a produção de novas folhas e as atividades reprodutivas frequentemente são suspensas, devido à realocação de nutrientes para produzir nova área fotossintética (ANTEN et al., 2003). Portanto, sugere-se proteção de indivíduos "porta sementes" (FREITAS \& PINARD, 2008), ou seja, poupar parte da população do extrativismo para que forneçam sementes em quantidade suficiente para a regeneração natural da espécie.

Além disso, a recuperação de grande área fotossintética é particularmente dificultosa, visto a baixa intensidade luminosa no interior da floresta, o que implica baixas taxas de produção foliar e de crescimento do estipe (CHAZDON, 1991e 1992). Essa limitação à luz sugere que estas palmeiras têm lenta recuperação frente às perdas, o que significa que monitoramentos em longo prazo são necessários para apurar com precisão os danos causados pela remoção de folhas em populações manejadas.

Sabendo-se que a área estudada já foi altamente impactada por extrativismo, sendo hoje local de coleta complementar, os valores de estoque natural em função da corrente demanda são preocupantes. As demais áreas ainda sujeitas à intensa coleta, em certo período de tempo ( $<15$ anos), podem vir a ter o mesmo cenário da área estudada. Nessa perspectiva, pode-se fazer o seguinte cálculo lógico: um extrator local geralmente retira, no mínimo, 1000 folhas por dia e, se em 0,5 ha foram registradas 448 folhas em ponto de corte, então seria necessário uma área de aproximadamente 1ha por dia para cada extrator atingir sua meta de corte. Mesmo que esse extrator venha a diversificar suas atividades e trabalhe apenas quatro dias por semana com o corte de palha, ainda haveria demanda de 16 ha por mês para cada um desses extratores. Na comunidade do Rasgadinho, moram 20 extratores, cuja atividade extrativista demandaria 320 ha por mês. Considerando-se o tempo de reposição foliar de oito meses (CECCONVALENTE, 2009), seriam necessários 2.560ha para os extratores poderem realizar rodízio e continuar fornecendo a quantia de 80.000 folhas por semana.

Nessa lógica, é bastante visível a situação eminente de alto impacto ambiental, tanto sobre o recurso alvo quanto seu entorno. Igualmente preocupante é o impacto socioeconômico para a comunidade extratora frente à decrescente disponibilidade do recurso em áreas cada vez mais longínquas.

\section{CONCLUSÃO}

O padrão de distribuição diamétrica $\mathrm{J}$ invertido registrado para a população de G. gamiova sugere uma população estável e auto-regenerativa. No entanto, os valores de estoque natural de folhas em função da demanda são preocupantes.

Registrou-se alta probabilidade da expressiva diminuição do recurso, assim como efeito deletério na população frente ao extrativismo, ação que necessita cuidadoso monitoramento. 


\section{REFERÊNCIAS}

ANTEN, N.P.R. et al. Defoliation and growth in an understory palm: quantifying the contributions of compensatory responses. Ecology, v.84, p.2905-2918, 2003. Disponível em: <http:// www.jstor.org/stable/3449961>. Acesso em: 17 set. 2012. doi: $10.1890 / 02-0454$

BALZON, D.R. Avaliação econômica dos produtos florestais não madeiráveis na área de proteção ambiental - APA de Guaratuba - Paraná. 2006. 195f. Tese (Doutorado em Ciências Florestais) - Setor de Ciências Agrárias, Universidade Federal do Paraná, Curitiba, PR

BAROT, S. et al. Demography of a savanna palm tree: predictions from comprehensive spatial pattern analyses. Ecology, v.80, n.6, p.1987-2005, 1999. Disponível em: <http://www.esajournals. org/doi/pdf/10.1890/0012-9658(1999)080\%5B1987\%3AD OASPT\%5D2.0.CO\%3B2>. Acesso em: 17 set. 2012. doi: 10.1890/0012-9658(1999)080[1987:DOASPT]2.0.CO;2.

BAULDAUF, C. Extrativismo de samambaia-preta (Rumohra adiantiformis (G.Forst) Ching) no Rio Grande do Sul: fundamentos para o manejo e monitoramento da atividade. 2006. 230f. Dissertação (Mestrado em Biologia Vegetal) - Universidade Federal de Santa Catarina, Florianópolis, SC.

CECCON-VALENTE, M.F. Subsídios ecológicos ao uso sustentável da palha Geonoma gamiova Barb. Rodr. (Arecaceae). 2009. 91f. Dissertação (Mestrado em Agronomia / Produção Vegetal) - Universidade Federal do Paraná, Curitiba, PR.

CHAZDON, R.L. Effects of leaf and ramet removal on growth and reproduction of Geonoma congesta, a clonal understorey Palm. Journal of Ecology, v.79, n.4, p.1137-1146, 1991. Disponível em: $<$ http://www.jstor.org/stable/2261104>. Acesso em: 17 set. 2012. doi: $10.2307 / 2261104$

CHAZDON, R.L. Patterns of growth and reproduction of Geonoma congesta, a clustered understory palm. Biotropica, v.24, p.43-51, 1992. Disponível em: <http://www.jstor.org/stable/2388472>. Acesso em: 17 set. 2012. doi: 10.2307/2388472.

FAO. Products florestales no madereros: posibilidades futures. Roma, 1992. 36p.

FAO. Non-wood forest products for rural income and sustainable development. Non-Wood Forest Products, n.7, p.1-138, 1995.

FREITAS, J.V.; PINARD, M. Applying ecological knowledge to decisions about seed tree retention in selective logging in tropical forests. Forest Ecology and Management, v.256, p.1434-1442, 2008. Disponível em: <http://www.sciencedirect.com/science/ article/pii/S0378112708002223 > . Acesso em: 17 set. 2012. doi: 10.1016/j.foreco.2008.03.001.

HENDERSON, A. et al. Field guide to the palms of the Americas. Princeton: Princeton University, 1997. 363p.

HOMMA, A.K.O. Extrativismo, biodiversidade e biopirataria na Amazônia. Brasília: EMBRAPA, 2008. 101p.

IAPAR. Cartas climáticas básicas do Estado do Paraná Londrina: IAPAR, 2000. CD-ROM.

KREBS, C.J. Ecological methodology. New York: Harper \& Row, 1989. 652p.

MOUSSOURIS, Y.; REGATO, P. Forest harvest: an overview of nom timber products in Mediterranean region. Roma: FAO, 1999. 25p.
NEGRELLE, R.R.B. Composição florística, estrutura fitossociológica e dinâmica de regeneração da floresta atlântica na Reserva de Vila Velha, município de Itapoá, SC. 1995. 222f. Tese (Doutorado em Ecologia) - Universidade Federal de São Carlos, São Carlos, SP.

REITZ, R. Palmeiras. In: REITZ, R. (Ed.). Flora ilustrada catarinense. Itajai: Herbário Barbosa Rodrigues, 1974. 180p.

RODERJAN, C.V. et al. As unidades fitogeográficas do estado do Paraná. Ciência \& Ambiente, n.24, p.75-92, 2002.

RODRIGUEZ-BURITICÁ, S. et al. Demography and life history of Geonoma orbignyana: na understory palm used as foliage in Colômbia. Forest Ecology and Management, v.211, p.329-340, 2005. Disponível em: <http://dx.doi.org/10.1016/j. foreco.2005.02.052>. Acesso em 17: set.2012. doi: 10.1016/j. foreco.2005.02.052

SCHMIDT, I.B. et al. Ethnobotany and effects of harvesting on the population ecology of Syngonanthus nitens (Bong.) Ruhland (Eriocaulaceae), a NTFP from Jalapão region, Central Brazil. Economic Botany, v.6, n.11, p.73-85, 2007. Disponível em: <http://download. springer.com/static/pdf/900/art\%253A10.1663\%252F0013$0001 \% 25282007 \% 252961 \% 255 \mathrm{~B} 73 \% 253 \mathrm{AEAEOHO} \% 255 \mathrm{D}$ 2.0.CO\%253B2.pdf?auth66=1352233102_6e4bddf882f67aba $5 \mathrm{~d} 9192 \mathrm{ce} 55 \mathrm{ffc} 04 \mathrm{~b} \& \mathrm{ext}=$.pdf $>$. Acesso em: 17 set. 2012. doi: 10.1663/0013-0001(2007)61[73:EAEOHO]2.0.CO;2.

SCOLFORO, J.R.S. et al. Modelagem da produção, idade das florestas nativas, distribuição espacial da espécies e análise estrutural. In: SCOLFORO, J.R.S. (Org.). Manejo florestal. Lavras: UFLA/FAEPE, 1998. p.189-246.

SONDA, C. Comunidades rurais tradicionais da área de proteção ambiental de Guaratuba: caracterização sócioeconômica e utilização dos recursos vegetais silvestres. 2002. 192f. Tese (Doutorado em Ciências Florestais) - Setor de Ciências Agrárias, Universidade Federal do Paraná, Curitiba, PR.

SOUZA, A.F.; MARTINS, F.R. Demography of the clonal palm Geonoma brevispatha in a Neotropical swamp forest. Austral Ecology, v.31, p.869-881, 2006. Disponível em: <http:// onlinelibrary.wiley.com/doi/10.1111/j.1442-9993.2006.01650.x/ pdf>. Acesso em: 17 set. 2012. doi: 10.1111/j.14429993.2006.01650.x

SVENNING, J.C.; MACIA, M.J. Harvesting of Geonoma macrostachys Mart. leaves for thatch: an exploration of sustainability. Restoration Ecology, v.167, p.251-262, 2002

SVENNING, J.C. Environmental heterogeneity, recruitment limitation and the mesoscale distribution of palms in a tropical montane rain forest (Maquipucuna, Ecuador). Journal of Tropical Ecology, v.17, p.97-113, 2001. Disponível em <http:// journals.cambridge.org/download.php?file $=\% 2$ F 10406 22DB951 9F93870673B0A9EB7A0AD34AE journals TRO TRO17 01 $\mathrm{S} 0266467401001067 \mathrm{a} . \mathrm{pdf} \&$ cover $=\overrightarrow{\mathrm{Y}} \& \operatorname{code}=\overline{7} 64 \mathrm{~b} 6 \overline{3} 0032 \mathrm{~d} 1 \overline{7} \mathrm{~b} 0 \overline{5}$ 968140 bc4db80943>. Acesso em 17 set. 2012. doi:do1:10.1017/ S0266467401001067.

VELOSO, H.P. et al. Classificação da vegetação brasileira adaptada a um sistema universal. Rio de Janeiro: IBGE, 1991. $123 \mathrm{p}$.

WEBB, L.S. et al. Regeneration and pattern in the subtropical rain forest. Journal of Ecology, v.60, p.675-695, 1972. Disponível em: $<$ http://www.jstor.org/stable/2258559>. Acesso em: 17 set. 2012. doi: $10.2307 / 225855$. 